\title{
The Rate Of Short Duration Gamma-Ray Bursts In The Local Universe
}

\author{
Soheb Mandhai ${ }^{* a}$ Nial Tanvir $^{a}$ Gavin Lamb $^{a}$ Andrew Levan $^{b, c}$ and David Tsang ${ }^{d}$, \\ ${ }^{a}$ Department of Physics and Astronomy, University of Leicester, University Road, LE1 7RH, U.K. \\ ${ }^{b}$ Department of Astrophysics/IMAPP, Radboud University, Nijmegen, The Netherlands \\ ${ }^{c}$ Department of Physics, University of Warwick, Coventry, CV4 7AL, U.K. \\ ${ }^{d}$ Department of Physics, University of Bath, Claverton Down, Bath, BA2 7AY, U.K. \\ E-mail: sfm13@leicester.ac.uk, nrt3@leiceseter.ac.uk, \\ gpl6@leicester.ac.uk, A. Levandastro.ru.nl, D. Tsangebath.ac.uk
}

\begin{abstract}
The binary neutron star merger responsible for the gravitational wave event, GW170817, strengthened the merger association with short-duration gamma-ray bursts (SGRBs) following the detection of the SGRB counterpart, GRB 170817A. Here we consider the constraints on a population of local gamma-ray bursts with moderately short duration $\left(T_{90}<4 \mathrm{~s}\right)$ and within $d<200 \mathrm{Mpc}$, that may have originated from similar compact binary mergers. Using well localised gamma-ray bursts from $\sim 14.5$ years of Swift/Burst Alert Telescope monitoring, we find no events with high likelihood of being in this distance range, and place an upper limit for the all-sky rate of such events of $<4 y^{-1}$. For Fermi/Gamma-ray Burst Monitor (GBM) and CGRO/Burst And Transient Source Experiment (BATSE) detected bursts, where the localisation has considerably larger uncertainties, we cross-correlated with 2MASS Redshift Survey galaxies at $d<100 \mathrm{Mpc}$, obtaining a weaker constraint of $<12 y^{-1}$. A separate correlation search from the GBM and BATSE bursts for giant flares originating from soft gamma-ray repeaters in nearby galaxies $(d<11 \mathrm{Mpc})$ yields an upper limit of $<3 y^{-1}$.
\end{abstract}

The New Era of Multi-Messenger Astrophysics - Asterics2019

25 - 29 March, 2019

Groningen, The Netherlands

${ }^{*}$ Speaker. 


\section{Overview}

In light of GRB 170817A that accompanied a gravitational wave detection of a merging neutron star binary at $\mathrm{d} \sim 40 \mathrm{Mpc}$, we consider the observational constraints on a nearby population of low-luminosity short-duration gamma-ray bursts (SGRBs), where we extend the definition to include bursts with $T_{90}<4 \mathrm{~s}$.

Compact binaries consisting of a neutron star paired with either a black-hole or another neutron star may receive natal kicks from the supernovae during formation [1]. Sufficiently high kick velocities can eject a binary from its host galaxy. Thus, although the majority of SGRBs (including GRB 170817A) occur within the bodies of their host galaxies in projection [2], binaries of this nature may merge at large separation from their host and hence their distance from us will not be determined. We consider two approaches to identifying potential nearby host galaxies associated with catalogued SGRBs, allowing for the possibility of such kicks. In Section 2, we determine the number of detected Neil Gehrels Swift Observatory (Swift) bursts that have tentative nearby host galaxies that fulfil our criteria. In Section 3, we describe a cross-correlation of catalogued SGRBs with galaxies within $100 \mathrm{Mpc}$.

\section{Using Swift to Localise Short-Duration Gamma-Ray Bursts}

Swift is a dedicated detection and follow-up satellite designed to observe gamma-ray bursts using the on-board Burst Alert Telescope (BAT) and their afterglows with the X-Ray Telescope (XRT) and UV/Optical Telescope (UVOT) [3]. Swift provides excellent localisation of bursts ranging from a few arcminutes, if observed only by BAT, to $0.5-5$ arcseconds, if observed by XRT or UVOT.

We searched for Swift SGRBs that lie within an on-sky projection distance of $200 \mathrm{kpc}$ (this distance corresponds to a long lived binary that has reasonable natal kick of $\sim 100 \mathrm{kms}^{-1}$ ) to galaxies within $d=200 \mathrm{Mpc}$ from the 2MASS Redshift Survey (2MRS) [4]. Table 1 lists the 10 bursts out of a total of $\sim 150$ Swift detected gamma-ray bursts with $\mathrm{T}_{90}<4 \mathrm{~s}$, that fulfill this criteria. None of these cases can be considered high confidence associations, and the total number is consistent with the expected rate of chance alignment of background bursts with foreground galaxies. We conclude an upper limit on the all-sky rate of local SGRBs to be $<4 y^{-1}$. Typical examples of bursts with either BAT or XRT error regions are shown in Figure 1.

\section{Correlating Fermi/GBM and CGRO/BATSE Gamma-Ray Burst locations with 2MRS Galaxies}

In comparison to Swift, the localisations from CGRO/BATSE and Fermi/GBM are considerably poorer but their fields of view are greater. As such, the joint BATSE+GBM population of observed gamma-ray bursts with $\mathrm{T}_{90}<4 \mathrm{~s}$ is larger at $\approx 800$. We performed a spatial crosscorrelation between these bursts and 2MRS galaxies (as described in [5], see also [13]) for the purposes of constraining the fraction of bursts that arise in galaxies within $100 \mathrm{Mpc}$. This yields a maximum upper limit on the all-sky rate of $\lesssim 12 y^{-1}$. An additional correlation using GBM and BATSE bursts with a $\mathrm{T}_{90}<1 \mathrm{~s}$ to constrain the soft gamma-ray repeaters (SGRs) giant flare rate in nearby galaxies $(d<11 \mathrm{Mpc})$, yielded a rate of $<3 y^{-1}$. 
Table 1: Compilation of Swift detected bursts that have candidate host galaxies within $200 \mathrm{Mpc}$ from the 2MASS Redshift Survey (2MRS). For other potential SGRB events that have tentative galactic hosts that are not present in 2MRS but have been discussed in literature are listed below the table break [5].

\begin{tabular}{|c|c|c|c|c|c|c|c|c|c|}
\hline GRB & $\begin{array}{l}T_{90} \\
(\mathbf{s})\end{array}$ & $\begin{array}{l}\text { Angular } \\
\text { Separa- } \\
\text { tion } \\
\text { (arcmin) }\end{array}$ & Closest Galaxy & $\begin{array}{l}\text { Galaxy } \\
\text { Type }\end{array}$ & $\begin{array}{l}\text { Optical } \\
\text { Bands } \\
(\mathbf{B} / \mathbf{R}) \\
(\mathbf{m a g})\end{array}$ & $\begin{array}{c}\mathrm{J}- \\
\text { Band } \\
\text { (mag) }\end{array}$ & $\begin{array}{c}d \\
(\mathbf{M p c})\end{array}$ & $\begin{array}{c}\text { Impact } \\
\text { Parame- } \\
\text { ter } \\
(\mathbf{k p c})\end{array}$ & $\begin{array}{c}E_{\text {iso }} \\
\left(10^{46} \text { ergs }\right.\end{array}$ \\
\hline 050906 & 0.26 & 2.0 & IC 0328 & $\mathrm{Sc}$ & 14.0 (B) & 12.2 & $132[6]$ & $77 \pm 109$ & 1.9 \\
\hline $100213 \mathrm{~A}$ & 2.40 & 5.4 & PGC 3087784 & SO-a & 14.7 (B) & 11.3 & $78[6]$ & 123 & 39.9 \\
\hline $111210 \mathrm{~A}$ & 2.52 & 6.0 & NGC 4671 & $\mathrm{E}$ & 13.4 (B) & 10.1 & $43[6]$ & 76 & 7.5 \\
\hline $120403 \mathrm{~A}$ & 1.25 & 4.9 & PGC 010703 & $\mathrm{Sc}$ & 14.4 (B) & 12.1 & $133[6]$ & $192 \pm 90$ & 38.2 \\
\hline $130515 \mathrm{~A}$ & 0.29 & 8.5 & PGC 420380 & SO-a & 16.0 (B) & 12.3 & $73[7]$ & 180 & 28.4 \\
\hline $160801 \mathrm{~A}$ & 2.85 & 6.7 & PGC 050620 & $\mathrm{Sa}$ & 15.2 (B) & 12.4 & $59[6]$ & 115 & 10.7 \\
\hline 181126A & 2.09 & 39.8 & NGC 3125 & E & 13.00 (B) & 11.3 & $14.9[8]$ & 173 & 1.9 \\
\hline 070809 & 1.30 & 2.0 & $\begin{array}{c}\text { PGC } 3082279 \\
{[9]}\end{array}$ & $\mathrm{Sa}$ & 16.3 (B) & 13.5 & $\begin{array}{c}180 \\
{[10]}\end{array}$ & 105 & 64.4 \\
\hline 090417A & 0.07 & 4.4 & $\begin{array}{c}\text { PGC } 1022875 \\
{[11]}\end{array}$ & SO-a & 15.9 (B) & 13.4 & $\begin{array}{c}360 \\
{[10]}\end{array}$ & $\begin{array}{c}461 \pm \\
292\end{array}$ & 24.5 \\
\hline $111020 \mathrm{~A}$ & 0.40 & 2.3 & FAIRALL 1160 & $\mathrm{Sa}$ & $\sim 14(\mathrm{R})$ & 11.7 & $81[12]$ & 54 & 9.4 \\
\hline
\end{tabular}

\section{In the Era of $\mathrm{LV}-\mathrm{O3}$}

So far, during the third LIGO/Virgo science run (LV-O3) there has been one detection (S190425z) with a high probability of originating from a neutron star binary merger within $200 \mathrm{Mpc}$. There were no detections of an accompanying gamma-ray transient reported, despite the localised field being observable by Fermi [14], and Integral [15].

\section{Acknowledgements}

We acknowledge use of the HyperLEDA; Extragalactic Distance Database; the NASA/IPAC Extragalactic Database. S.F.M. is supported by a PhD studentship funded by the College of Science and Engineering at the University of Leicester; G.P.L. is supported by STFC grants; N.R.T. and A.J.L. acknowledge support from ERC Grant 725246 TEDE.

\section{References}

[1] J. C. Bray and J. J. Eldridge, Neutron star kicks and their relationship to supernovae ejecta mass, 461 (2016) 3747.

[2] W. Fong, E. Berger, R. Chornock, R. Margutti, A. J. Levan, N. R. Tanvir et al., Demographics of the Galaxies Hosting Short-duration Gamma-Ray Bursts, 769 (2013) 56.

[3] N. Gehrels, G. Chincarini, P. Giommi, K. Mason, J. Nousek, A. Wells et al., The swift gamma-ray burst mission, The Astrophysical Journal 611 (2004) 1005. 
[4] J. P. Huchra, L. M. Macri, K. L. Masters, T. H. Jarrett, P. Berlind, M. Calkins et al., The 2MASS Redshift Survey - Description and Data Release, 199 (2012) 26.

[5] S. Mandhai, N. Tanvir, G. Lamb, A. Levan and D. Tsang, The rate of short-duration gamma-ray bursts in the local universe, Galaxies 6 (2018) 130.

[6] D. Makarov, P. Prugniel, N. Terekhova, H. Courtois and I. Vauglin, HyperLEDA. III. The catalogue of extragalactic distances, 570 (2014) A13.

[7] R. B. Tully, H. M. Courtois and J. G. Sorce, Cosmicflows-3, 152 (2016) 50.

[8] R. B. Tully, L. Rizzi, E. J. Shaya, H. M. Courtois, D. I. Makarov and B. A. Jacobs, The Extragalactic Distance Database, 138 (2009) 323.

[9] D. A. Perley, J. S. Bloom, M. Modjaz, A. A. Miller, J. Shiode, J. Brewer et al., GRB 070809: putative host galaxy and redshift., GRB Coordinates Network 7889 (2008).

[10] G. Helou, B. Madore, M. Schmitz, M. Bicay, X. Wu and J. Bennett, The nasa/ipac extragalactic database, in Databases \& On-Line Data in Astronomy, pp. 89-106, Springer, (1991).

[11] P. T. O'Brien and N. R. Tanvir, GRB 090417A: nearby galaxy redshift., GRB Coordinates Network 9136 (2009) .

[12] R. Tunnicliffe, A. J. Levan, N. R. Tanvir, A. Rowlinson, D. Perley, J. Bloom et al., On the nature of the 'hostless' short grbs, Monthly Notices of the Royal Astronomical Society 437 (2013) 1495.

[13] N. R. Tanvir, R. Chapman, A. J. Levan and R. Priddey, An origin in the local universe for some short $\gamma$-ray bursts, 438 (2005) 991.

[14] C. Fletcher, LIGO/Virgo S190425z: Fermi GBM Observations, GRB Coordinates Network 24185 (2019) .

[15] A. Martin-Carillo, V. Savchenko, C. Ferrigno, J. Rodi , A. Coleiro and S. Mereghetti, LIGO/Virgo S190425z: INTEGRAL prompt observation, GRB Coordinates Network 24169 (2019) .

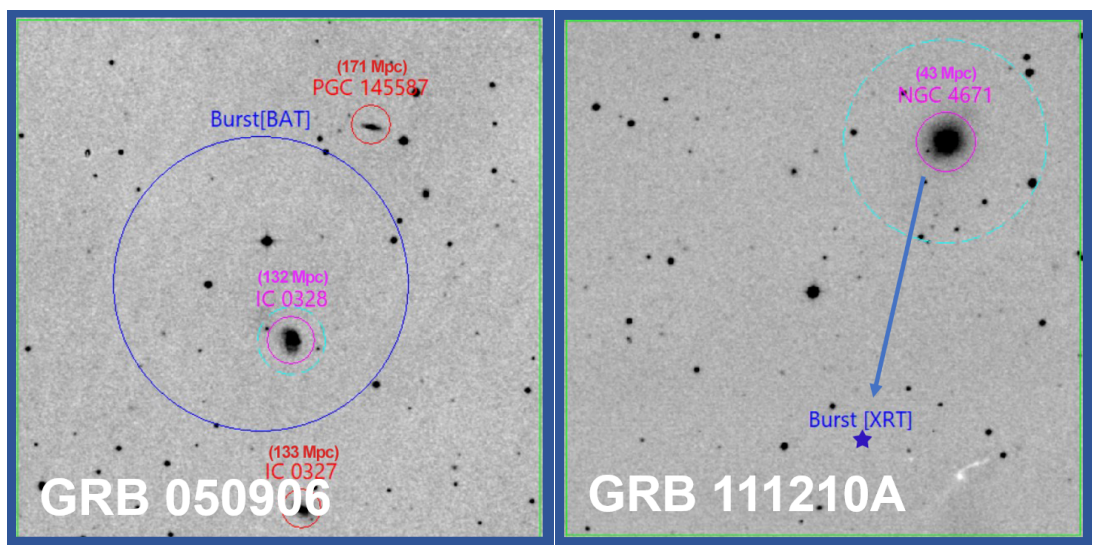

Figure 1: Potential galactic hosts to SGRB events that are within a projected distance of $200 \mathrm{kpc}$ from the burst's location. [Left panel] An example case using GRB 050906, where a potential host, IC 0328, appears within the BAT uncertainty region. For this example, no X-ray or optical counterpart was identified. [Right panel] An example case of GRB 111210A, where a potential host galaxy (NGC 4671) is found at a displaced distance from the XRT localisation of the burst. In each case, the dashed cyan circle corresponds to a $25 \mathrm{kpc}$ offset from the candidate host [5]. 